\section{Abschließende Bemerkungen}

Wie zahlreiche Vorträge zeigten, hatte als jüngere theoretische Entwicklung im Feld der TM für die diesjährige Sommerakademie Geels MehrEbenen-Perspektive (Geels 2002) eine herausragende Bedeutung. Die damit verbundene Vorstellung von Transitions als Innovationspfade und deren Durchlaufen der drei zuvor beschriebenen Ebenen war für viele Diskussionen leitend, die Nützlichkeit des Ansatzes im Sinne einer gemeinsamen Heuristik sei hier deshalb betont. Um jedoch als Theorie zur Erklärung von Innovationen beitragen zu können, ist - im Sinne der Kritik von Genus - eine grundlegende Ausarbeitung des Ansatzes notwendig.

Mit den hier vorgestellten Vorträgen wurde sicherlich nur eine von vielen Diskussionslinien der Sommerakademie nachgezeichnet, unerwähnt blieb beispielsweise die Präsentation einer Vielzahl von Fallstudien, in denen neben nationalen oder regionalen institutionellen Strukturen auch konkrete Technologien thematisiert wurden. Vor allem die vielen Doktorandenvorträge, die rund die Hälfte aller Präsentationen ausmachten, gaben der Sommerakademie einen sehr offenen, lebhaften und produktiven Charakter.

\section{Literatur}

Carlsson, B.; Stankiewicz, R., 1991: On the Nature, Function and Composition of Technological Systems. Journal of Evolutionary Economics 1/2 (1991), pp. 93-118

Geels, F.W., 2002: Technological Transitions as Evolutionary Reconfiguration Processes: A MultiLevel Perspective and a Case-Study. In: Research Policy 31/8-9 (2002), pp. 1257-1274

Nelson, R.R.; Winter, S.G., 1982: An Evolutionary Theory of Economic Change. Cambridge, MA

Rip, A.; Kemp, R., 1998: Technological Change. In: Rayner, S.; Malone, E.L. (eds.): Human Choice and Climate Change. Columbus, Ohio, pp. 327-399

Rotmans, J.; Kemp, R.; van Asselt, M. et al., 2000: Transitions \& Transition Management. For a Low Emission Energy Supply. A Study for the $4^{\text {th }} \mathrm{Na}$ tional Environmental Policy Plan (NMP4). Maastricht; http://www.icis.unimaas.nl/projects/nmp4/ downs/00_35_ab.pdf

)

\section{Integrating Natural and Social Sciences for Sustainability} Tagungsbericht von der ", $7^{\text {th }}$ International Conference of the European Society for Ecological Economics"

\section{Leipzig, 5. - 8. Juni 2007}

\section{von Jürgen Kopfmüller, ITAS, und Fred Luks, Österreichisches Institut für Nachhaltige Entwicklung, Wien}

Unter dem Titel „Integrating Natural and Social Sciences for Sustainability" lud die Europäische Gesellschaft für Ökologische Ökonomie (European Society for Ecological Economics - ESEE) vom 5. bis 8. Juni $2007 \mathrm{zu}$ ihrer siebten internationalen Konferenz ins Helmholtz-Zentrum für Umweltforschung (UFZ) nach Leipzig ein. Die Veranstaltung, an der rund 500 Personen vorwiegend WissenschaftlerInnen - aus der ganzen Welt teilnahmen, wurde gemeinsam von der ESEE, dem UFZ, der Vereinigung für Ökologische Ökonomie (VÖÖ) und der Vereinigung für Ökologische Wirtschaftsforschung (VÖW) organisiert. ${ }^{1}$

Der Tagungstitel „Integrating Natural and Social Sciences for Sustainability" gibt einerseits das Selbstverständnis der Ökologischen Ökonomie als einer inter- und transdisziplinär wirkenden Nachhaltigkeitswissenschaft wieder. So versteht sich die ESEE nicht nur als Vereinigung von ÖkonomInnen, sondern als eine wissenschaftliche Organisation, die disziplinenübergreifend das Nachhaltigkeitsleitbild erforschen und seine Umsetzung voranbringen will. Andererseits bringt der Tagungstitel den Integrationsanspruch einiger WissenschaftlerInnen bzw. die Integrationsforderung an die Wissenschaft selbst zum Ausdruck, die der Beobachtung geschuldet ist, dass Natur- und Sozialwissenschaften nach wie vor kaum integrativ zusammenwirken, sondern im Gegenteil oft unvermittelt nebeneinander stehen.

$\mathrm{Ob}$ die Veranstaltung und die dort präsentierten Beiträge diesen sehr hohen Anspruch der Integration wirklich erfüllen konnten, ist nicht zuletzt eine Frage der Perspektive. Zu beobachten war jedenfalls auch hier, dass BiologInnen eben hauptsächlich biologisch argumentieren, ÖkonomInnen im Wesentlichen wirtschaftlich 
denken und sprechen und so weiter. Gleichwohl waren auf dieser Tagung überdurchschnittlich viele Beiträge zu hören, bei denen zumindest das ehrliche Bemühen und oft auch ein erfolgreiches Umsetzen von disziplinenübergreifendem Arbeiten, das bereits bei der Konzipierung von Forschungsprojekten beginnt, erkennbar waren. Ein wesentlicher (Mehr)Wert dieser Veranstaltung war sicher, über die weltweite Existenz solcher Aktivitäten und deren Ergebnisse überhaupt Kenntnis zu erlangen und entsprechende Kontakte knüpfen zu können.

Positiv anzumerken ist auch, dass es den Organisatoren gelungen ist, mit dem Angebot unterschiedlicher Präsentations- und SessionFormen einen vielfältigen, abwechslungsreichen und relativ entzerrten Verlauf der insgesamt dreieinhalb Tage zu realisieren. Beispielsweise wurden so genannte „Core Sessions“ durchgeführt, in denen neben Präsentationen vorbereitete Kommentare von Ko-Referenten vorgetragen und diskutiert wurden. Daneben gab es so genannte „Short Paper Sessions“, die aus Kurzpräsentationen bestanden, was in der Regel mehr Raum für Diskussionen ließ. Damit war es den Veranstaltern auch möglich gewesen, qualitative Abstufungen bei der Annahme von eingereichten Papieren vorzunehmen.

Des Weiteren wurden Symposien durchgeführt, bei denen es auf Einladung der jeweiligen Sitzungsleitung Präsentationen $\mathrm{zu}$ bestimmten Themen gab. Es ging dabei um grundlegende Forschungsthemen wie „Management of complex commons“, „Economics and Sustainability" oder "The Philosophy of Ecological Economics", aber auch um konkretere Aspekte wie „Global change impacts on the Elbe river basin“, „Sustainable urban development” oder „Integrating ecological and economic knowledge in models for biodiversity management". Schließlich wurde der Kurzpräsentation von Postern und der Diskussion mit deren Autoren relativ viel Raum und Zeit gewidmet, sie erhielten damit mehr Aufmerksamkeit als häufig bei derartigen Veranstaltungen üblich.

Neben diesen Präsentationsvarianten gab es natürlich auch Plenarsitzungen - üblicherweise Höhepunkte von Konferenzen. Solche „Plenaries“ dienen einerseits dazu, mit möglichst prominenten Rednern für Aufmerksamkeit zu sorgen. Anderseits bieten diese Veranstaltungen häufig auch Hinweise darauf, was gerade in der Community ,angesagt“ ist. Legen wir diesen Indikator zu Grunde, dann gilt: Angesagt ist ein hohes Selbstbewusstsein bei den Ökologischen Ökonomen, ein in Konturen erkennbares neues Wissenschaftsverständnis und mit Abstrichen auch nach wie vor die $\mathrm{Ab}$ grenzung gegenüber dem (wirtschafts)wissenschaftlichen Mainstream. Was den letzten Punkt betrifft, war der Plenarvortrag ,Living in a time of revolutions - The Struggle for a New Economic Order" von Clive Spash (Universität Canberra) sicher die prägnanteste Stimme. Spash präsentierte seine Kritik der herrschenden Ökonomik mit kämpferischen, bisweilen gar „kriegerischen“ Metaphern.

Auch der Plenarbeitrag von Inge Røpke (Technical University of Denmark, Copenhagen) bot mit Gedanken über die Nachhaltigkeitsfolgen von Freizeitverhalten (zum Beispiel auch von NachhaltigkeitsforscherInnen!) einige originelle Einsichten. Im Hinblick darauf, wohin sich eine problemorientierte Nachhaltigkeitswissenschaft in Zukunft entwickeln könnte, war der Beitrag von Richard Norgaard (University of California, Berkeley) einer der interessantesten. Norgaard berichtete unter der Überschrift „Collectively Understanding Socio-ecological Systems“ darüber, wie im Rahmen des „Millennium Ecosystem Assessment Projects" auf diskursive Weise nachhaltigkeitsrelevantes Wissen erzeugt wird. Damit beschrieb er sozusagen einen der Leitfäden der Konferenz, war doch derartig erarbeitetes Wissen Gegenstand vieler Beiträge. Malte Faber (Emeritus der Universität Heidelberg), in den 1980er Jahren einer der ersten Umweltökonomen in Deutschland, beschrieb in seinem Plenarvortrag seinen persönlichen Weg zur Ökologischen Ökonomie, die ihn dabei begleitenden Schwierigkeiten und gewonnenen Einsichten und seine Schlussfolgerungen hinsichtlich der künftigen Perspektiven der Ökologischen Ökonomie.

Elinor Ostrum (Indiana University, Bloomington) und Christian Hey (Geschäftsführer des Sachverständigenrats für Umweltfragen in Deutschland $<$ SRU $>$ ) berichteten schließlich über die Möglichkeiten, Elemente von Interund Transdisziplinarität in die Politik bzw. die Politikberatung einzubringen und welche Ergebnisse aus ihrer Sicht dabei bislang zu verzeichnen seien. Ostrum fokussierte dabei, vor dem Hintergrund ihrer langjährigen Erfahrun- 
gen in internationalen Forschungsprojekten, zum einen auf die Bedeutung von Institutionen bzw. institutionellen Bedingungen für die interdisziplinäre Erarbeitung von Beratungswissen, zum anderen auf die Möglichkeit, insbesondere unter den Bedingungen lokaler und regionaler Politik- und Entscheidungsprozesse nicht-wissenschaftliche Akteure in die Wissenserzeugung angemessen einbeziehen $\mathrm{zu}$ können. Hey bezog sich insbesondere auf die Erfahrungen mit der, in der Tat, relativ breiten interdisziplinären Zusammensetzung des SRU und auf die Möglichkeiten, bei der Vergabe von Auftragsarbeiten und -gutachten eine, den Themenfeldern angemessene, interdisziplinäre Herangehensweise gewährleisten zu können. Die Einsichten dieser beiden Experten aus unterschiedlicher Praxisperspektive waren teilweise ermutigend, teilweise gaben sie aber auch Anlass zu Ernüchterung und förderten die Erkenntnis, dass hier noch viel zu tun bleibt.

In der nachhaltigkeitsrelevanten Politikberatung bestünde im Prinzip ein weites Betätigungsfeld für die Ökologische Ökonomie, die sich von Anbeginn als inter- und transdisziplinärer Forschungsansatz verstanden und dies auch unter Beweis gestellt hat. Damit kann sie zumindest den Anspruch erheben, eher als traditionelle Ansätze geeignet zu sein, die Anforderungen an eine derartige Beratung erfüllen zu können (dieser Einwurf sozusagen ,in eigener Sache" sei hier gestattet). Einige Beiträge der Konferenz haben hier bereits erste Ansätze offenbart, vielfach ist jedoch die Hemmschwelle leider noch recht hoch, sich mit diesem Forschungsansatz in das Feld der Politikberatung zu begeben.

Insgesamt waren die Beiträge der Veranstaltung durch eine große inhaltliche Bandbreite geprägt. Angesichts des Tagungsthemas verwundert dies nicht. Vielmehr macht es deutlich, dass sich die Community der Ökologischen Ökonomie und diejenigen, die ihr mehr oder weniger nahe stehen, mittlerweile mit der Nachhaltigkeitsthematik in ihrer ganzen Breite auseinandersetzen. Ein gutes Zeichen. Der Versuch, sich der Herausforderung zu stellen, diese Breite im Rahmen einer einzigen Tagung darzustellen, hat jedoch auch einen Preis. An drei Tagen fanden rund 80 Sessions mit über 300 Präsentationen statt. Das Organisationsteam des UFZ bewältigte diese Aufgabe hervorragend. Allerdings birgt eine solche Mammutveranstaltung zum einen die Gefahr, dass die Teilnehmer durch ein zu großes Angebot in zu kurzer Zeit ,erschlagen" werden; zum anderen war es angesichts der Tatsache, dass in der Regel 10 Sessions parallel liefen, selbst bei bestem Willen und bester körperlicher Kondition leider nur möglich, einen Bruchteil des Angebotenen wahrzunehmen.

Neben diesem quantitativen war auch das qualitative Niveau der allermeisten von den Autoren besuchten Sessions beachtlich hoch. Nachfragen bei anderen Teilnehmern zu den übrigen Veranstaltungen ergaben häufig eine ähnliche Einschätzung. Gleichwohl wurde man in einzelnen Sessions auch mit Beiträgen konfrontiert, deren Qualität als durchaus fragwürdig einzustufen war. Hierin kommt - wie im Prinzip bei allen großen Tagungen - auch das Spannungsfeld zwischen dem Anspruch der Gewährleistung wissenschaftlicher Qualität und dem ökonomischen Zwang zur Kostendeckung durch eine hohe Teilnehmerzahl zum Ausdruck, in dem sich Organisatoren solcher Veranstaltungen stets bewegen müssen.

Aus Sicht der Berichterstatter ist bei dieser Konferenz der schwierige Spagat zwischen Größe und Qualität alles in allem gut geglückt, da bei den meisten TeilnehmerInnen die positiven Effekte der erhaltenen Anregungen und Kontaktmöglichkeiten sowie der gewonnenen Erkenntnisse die kritischen Eindrücke deutlich überwogen haben dürften.

\section{Zu den Autoren}

Jürgen Kopfmüller ist Vorsitzender der Vereinigung für Ökologische Ökonomie e.V. (VÖÖ); er war Mitglied des Reviewer Gremiums der Konferenz.

Fred Luks war bis 2007 ebenfalls Vorsitzender der VÖÖ; er war Mitglied im Local Organizing Committee und im Scientific Committee der Konferenz.

\section{Anmerkungen}

1) Ausführliche Informationen zur Konferenz und ihren Veranstaltern finden Sie auch auf der Projekt-Homepage http://www.esee2007.ufz.de/. 